\title{
SISTEMA DE IDENTIFICACIÓN DE OBJETOS PARA PERSONAS INVIDENTES USANDO LA TECNOLOGÍA RFID
}

\author{
Luis Enrique Ortiz Fernández ${ }^{1, *}$
}

\section{Resumen}

En Ecuador existen más de trescientos mil invidentes, cifra que crece cada día. La mayoría de estas personas no tienen independencia de elección en muchas situaciones, siendo una de las más comunes no poder elegir los objetos que deben comprar en algún comercio. En este artículo se describe el diseño e implementación de un sistema que permite identificar objetos mediante la reproducción de una descripción auditiva de los mismos, proporcionando a las personas invidentes la posibilidad de mejorar su calidad de vida. La tecnología base para el funcionamiento del sistema es la RFID - Identificación por Radio Frecuencia. El sistema consta de un dispositivo móvil desarrollado a partir de varios componentes electrónicos y de un software para PC implementado en Visual C\# 2010 Express. Los requisitos de diseño fueron definidos mediante una encuesta aplicada a una muestra de la población invidente en la ciudad de Cuenca. Concluida la implementación se realizaron pruebas técnicas y de satisfacción del sistema, en varios centros de ayuda a personas invidentes.

Palabras clave: audio MP3, bases de datos SQL, identificación de objetos, invidente, lenguajes C, PIC32, RFID.

\begin{abstract}
In Ecuador there are over three hundred thousand blind people a number that grows every day. Most of these people have no independence of choice in many situations being one of the most common cannot choose objects that they should buy in some trade. This paper describes the design and implementation of a system which identifies objects through of the playing an auditory description of these giving blind people the opportunity to improve their quality of life. The basis technology for the operation of the system is the RFID - Radio Frequency Identification. The system consists of a mobile device developed from various electronic components and PC software implemented in Visual C \# 2010 Express.The design requirements were defined through of a survey applied to one sample of the blind people population in the city of Cuenca. Concluded the implementation were realized the techniques testing and of satisfaction of the system, in several centers helps blind people.
\end{abstract}

Keywords: audio MP3, SQL databases, object ID, blind, C, languages, PIC32, RFID.

\footnotetext{
$\overline{1, *}$ Ingeniero Electrónico, Diseñador y desarrollador de soluciones RFID. Autor para correspondencia : leofaf@gmail.com Recibido: 26 - Septiembre - 2012, Aprobado tras revisión: 16 - Noviembre - 2012

Forma sugerida de citación: Ortiz Fernández, L. (2012). "Sistema de identificación de objetos para personas invidentes usando la tecnología RFID". INGENIUS. N. ${ }^{\circ} 8$, (Julio/Diciembre). pp. 38-44. ISSN: 1390-650X
} 


\section{Introducción}

En Ecuador existen 39160 personas invidentes registradas en el CONADIS-Consejo Nacional de Discapacidades [1].

Para mejorar la situación de las personas con discapacidad visual, hace falta desarrollar herramientas tecnológicas que les permitan desenvolverse de forma independiente en sus hogares, trabajo, etc.

Conforme ha evolucionado la electrónica e informática, se han desarrollando tecnologías como la RFIDIdentificación por Radiofrecuencia - Radio Frequency Identification, cuyo objetivo básico es el almacenamiento y recuperación de datos a través de ondas de radio desde dispositivos denominados tag's (etiquetas, llaveros, botones, etc).

Debido a que cada etiqueta RFID posee un número único e irrepetible y a que no requieren línea de vista ni contacto físico con el lector, esta tecnología puede ser empleada para el desarrollo de aplicaciones de asistencia a personas con discapacidad visual.

Juntando etiquetas [2] y un lector RFID [3] con dispositivos electrónicos como el Mikromedia PIC32 [4] y los paquetes de software Visual TFT [5], MikroC Profor PIC32 [6] y Microsoft Visual C\# 2010 Express [7], se ha desarrollado un sistema IOI - Identificación de Objetos para Invidentes.

Este sistema posee dos partes, un dispositivo portátil que permite a los invidentes reconocer objetos al escuchar una descripción de los mismos y de un software que ayuda a los videntes a interconectar el dispositivo con un PC, recibir el ID-Identificador-Identifier de una tag RFID, grabar un archivo de audio MP3, verificar el audio mediante un reproductor y administrar una base de datos SQL Lenguaje de Consulta Estructurado Structured Query Language para guardar información acerca de los objetos etiquetados.

El principal objetivo del sistema IOI es mejorar la calidad de vida de las personas invidentes e inmiscuir en este proceso a todas las personas que están a su alrededor. Se busca implementar el sistema en centros comerciales para facilitar las tareas de compra y venta de este sector vulnerable de la sociedad.

\section{Descripción y funcionamiento del sistema IOI}

Es un equipo electrónico que permite a los invidentes reconocer objetos al escuchar una descripción de los mismos.

Este dispositivo fue diseñado y desarrollado de acuerdo a los siguientes requisitos:
- Manejar con un solo microcontrolador varios módulos multimedia.

- Tener módulos que permitan leer (lector RFID) y comprobar el ID de las etiquetas RFID, leer y escribir tarjetas de memoria microSD, reproducir archivos MP3 a través de audífonos y un módulo de comunicación serial para conectar el dispositivo a un PC.

- Poseer dos modos de funcionamiento, administrador y usuario.

- Tener una fuente de energía propia, mediante batería o pilas.

Tomando en cuenta los requisitos de diseño se eligió como hardware base al equipo Mikromedia PIC32 debido a que incorporaba los módulos necesarios para desarrollar el dispositivo IOI y su precio era asequible.

Para realizar la interfaz gráfica del dispositivo IOI se escogió Visual TFT porque permite crear interfaces gráficas para dispositivos Mikromedia PIC32 y su IDE - Entorno de Desarrollo Integrado - Integrated Development Environment es amigable con el desarrollador.

Para programar los algoritmos que hacen funcionar el dispositivo IOI se escogió el compilador MikroC Pro for PIC32 ya que proporciona librerías para todos los módulos del Mikromedia PIC32.

En la Figura 1 se observan los módulos y elementos del hardware del dispositivo. Al presionar el botón "Administrador", el dispositivo IOI realiza las siguientes tareas:

- Leer y comprobar el ID de la etiqueta RFID.

- Codificar el ID en un número entero de 8 bits.

- Enviar el ID codificado a una aplicación para PC, mediante comunicación serial o USB.

Por otra parte cuando se presiona "Usuario" el dispositivo hace lo siguiente:

- Leer y comprobar el ID de la etiqueta RFID.

- Codificar el ID en un número entero de 8 bits.

- Buscar dentro de la tarjeta de memoria microSD un archivo de audio que lleve por nombre el ID codificado y reproducirlo a través de audífonos.

El dispositivo final consta de los componentes que se muestran en la Figura 2; posee dos pilas AAA, una batería, una memoria microSD, un adaptador USBSerial y audífonos. 


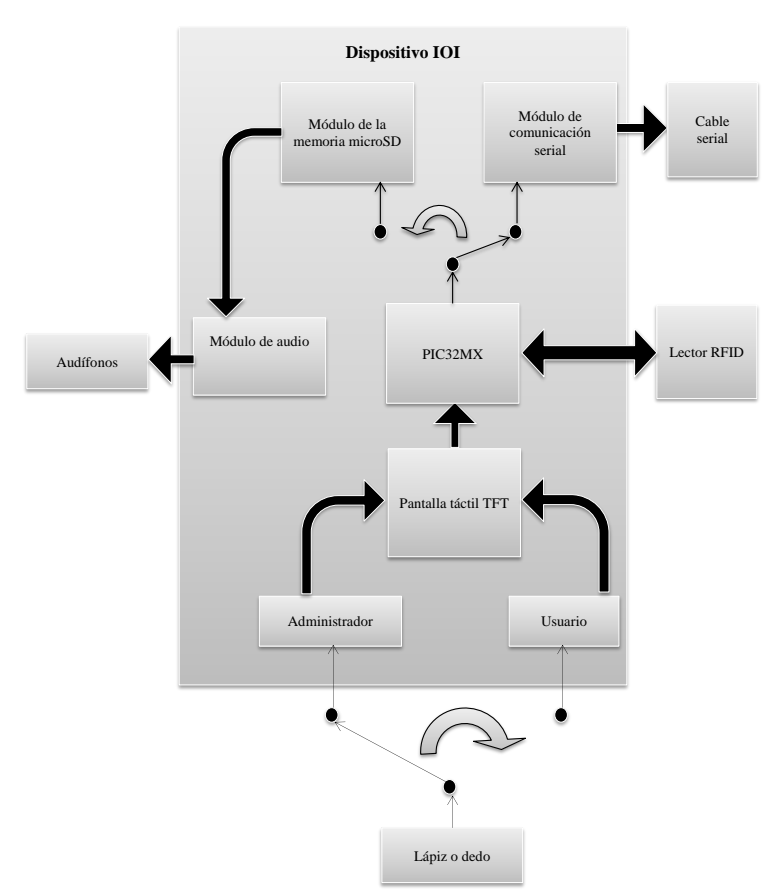

Figura 1. Diagrama de bloques del hardware del dispositivo IOI.

Terminada la implementación del hardware se desarrollaron los algoritmos para el dispositivo. (Ver Figura 3).

El diagrama de flujo del código realizado en MikroC Pro for PIC32 se muestra en la Figura 4. En este diagrama se encuentran funciones como "Leer_ID_RFID" que permite al PIC32 recibir y comprobar el ID RFID de las etiquetas, y determinar si asociar el ID a un archivo de audio en caso de que se haya presionado el botón "Usuario" o enviarlo a un PC a través del módulo de comunicación serial en caso de que se haya presionado el botón "Administrador".

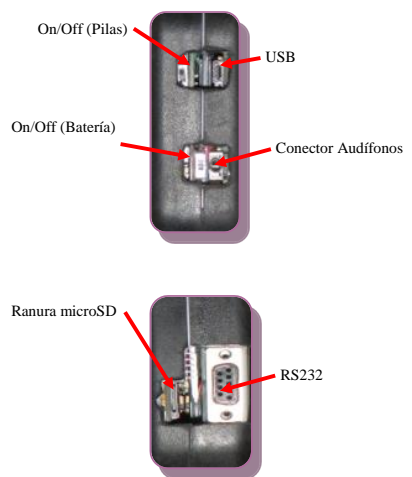

(a)

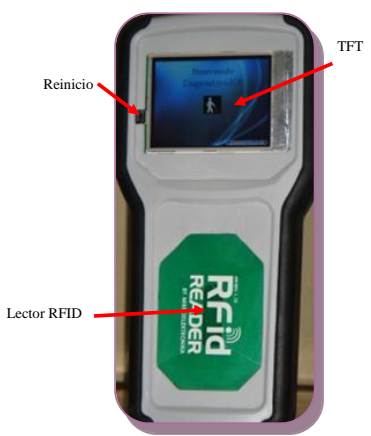

(b)
Figura 2. Dispositivo IOI final. (a) Vistas laterales (b) Vista frontal

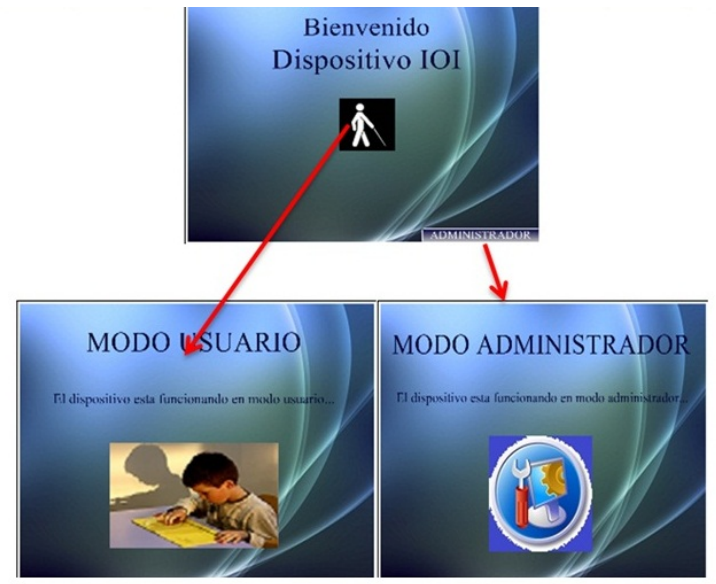

Figura 3. Interfaz gráfica para el dispositivo IOI creada en Visual TFT.

\section{$2.1 \quad$ Software IOI}

Es una aplicación creada para el administrador del sistema IOI, es compatible con Windows XP, Vista y 7 de 32 bits; permite realizar lo siguiente:

- Conectar el dispositivo IOI a un PC, de forma serial o USB.

- Leer el ID de una etiqueta RFID (pasiva de $125 \mathrm{KHz}$ ) adherida a cualquier objeto.

- Grabar archivos MP3 con un volumen y tasa de bits ajustable.

- Reproducir archivos MP3.

- Manejar una base de datos de SQL y almacenar información adicional acerca de los objetos que posean una etiqueta RFID.

Para implementar el software IOI se usó Visual C\# 2010 Express por ser la plataforma de desarrollo para Windows más eficaz ya que permite manejar bases de datos y es gratuito.

Luego de la programación se obtuvo la aplicación de la Figura 5 que consta de varios paneles.

En la Figura 6 se ve el panel 1, que posee las herramientas para establecer la conexión y recibir los datos enviados por el dispositivo IOI.

En la Figura 7 se muestra el panel 2, que permite grabar archivos MP3 y guardarlos con el nombre del ID de una etiqueta RFID para que el prototipo pueda asociar el archivo de audio a la etiqueta adherida a un objeto o producto.

En el panel 3 (ver Figura 8) el administrador puede manejar una base de datos de SQL con una capacidad de almacenamiento de hasta $10 \mathrm{~GB}$, esta base de datos 


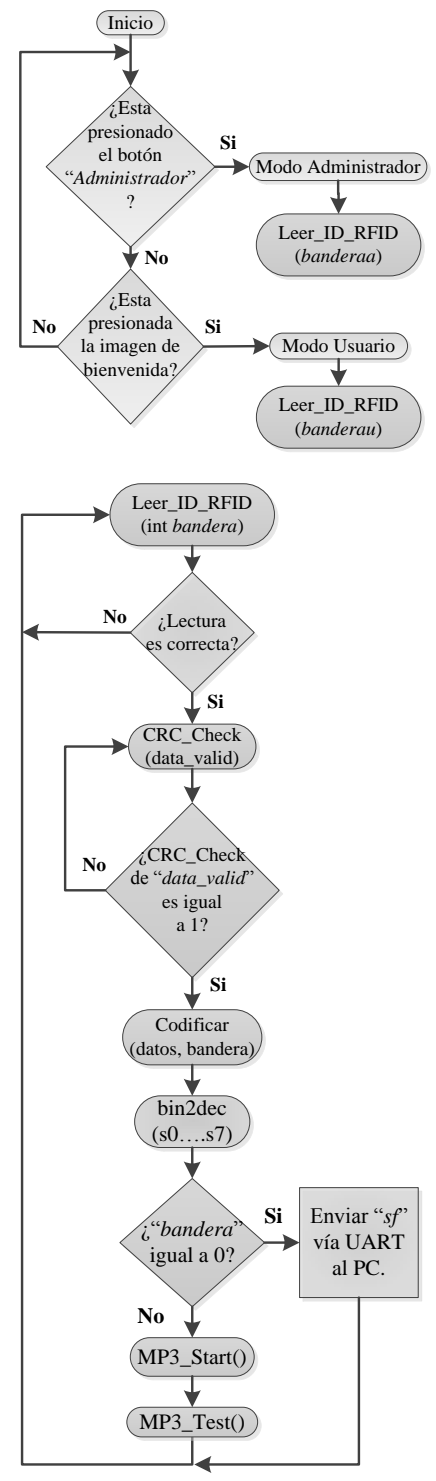

Figura 4. Diagrama de flujo del algoritmo para el dispositivo IOI implementado en MikroC PRO for PIC32.

esta conformada por una tabla que crece dinámicamente y se puede guardar información más detallada sobre el objeto que tiene adherido una etiqueta RFID.

\subsection{Administrador}

Es la persona que administra el sistema usando el dispositivo en modo administrador y el software IOI, sus funciones son (ver Figura 9):

- Adherir las etiquetas RFID en los objetos o productos que el usuario desee.

- Encender, conectar y seleccionar el modo administrador del dispositivo IOI.

- Con la ayuda del software IOI registrar el ID

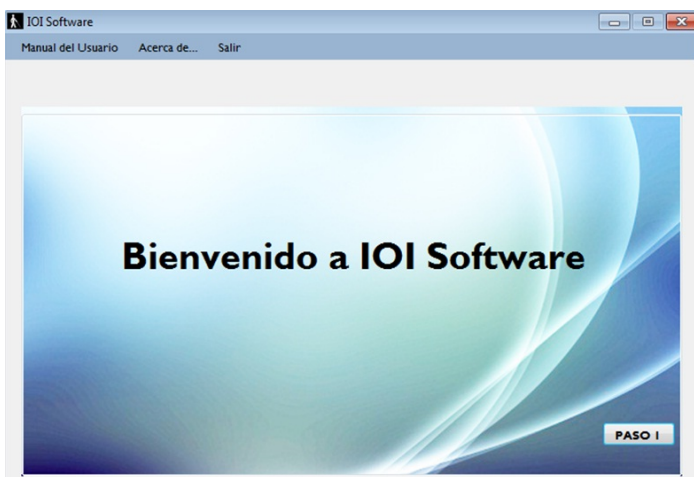

Figura 5. Menú inicio software IOI.

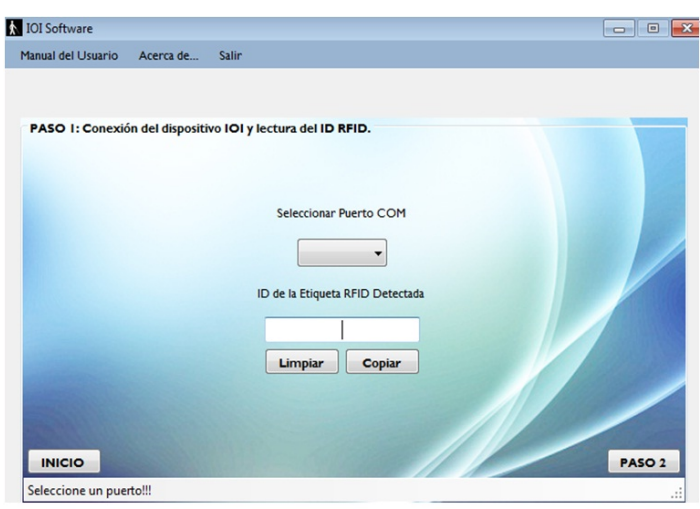

Figura 6. Panel 1 del software IOI.

de la etiqueta RFID, grabar el audio MP3 con la descripción del objeto o producto, guardarlo en la memoria microSD y finalmente ampliar y guardar la información de los objetos o productos usando una base de datos.

\section{$2.3 \quad$ Usuario}

Es la persona invidente que usa el dispositivo IOI en modo usuario para identificar objetos, sus funciones son (ver Figura 10):

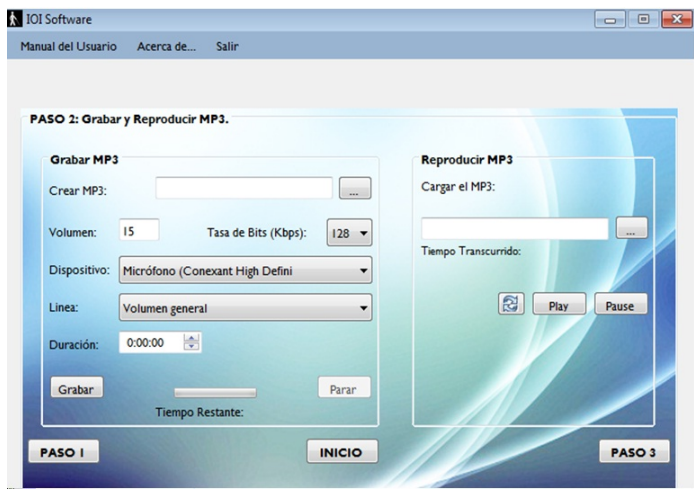

Figura 7. Panel 2 del software IOI. 


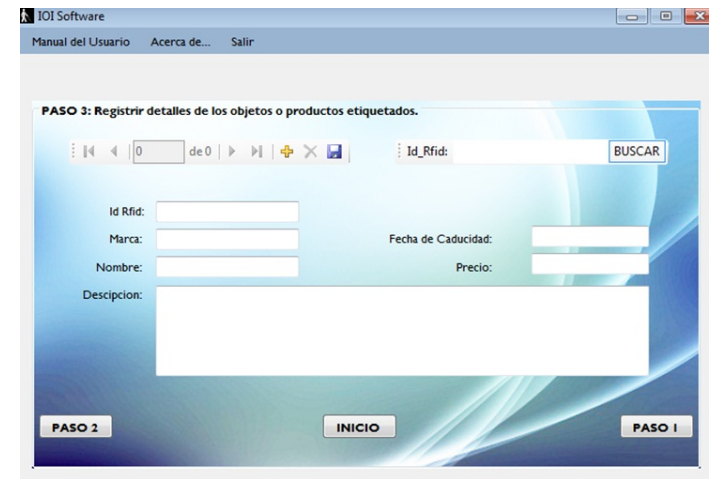

Figura 8. Panel 3 del software IOI.

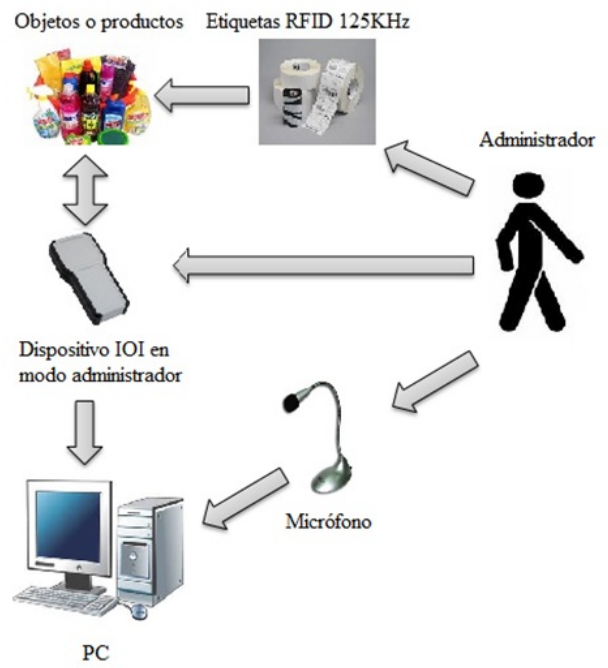

Figura 9. Funciones del administrador dentro del sistema IOI.

- Encender el dispositivo y seleccionar el modo usuario.

- Colocarse los audífonos.

- Acercar el dispositivo a un objeto o producto y escuchar su descripción.

\section{Pruebas aplicadas al dispositivo y software IOI}

Para realizar las pruebas se contó con la participación de personas miembros de las instituciones IEISA - Instituto Fiscal Especial de Invidentes y Sordos del Azuay, FENCE - Federación Nacional de Ciegos del Ecuador y SONVA-Sociedad de No Videntes del Azuay (ver Figura 11).

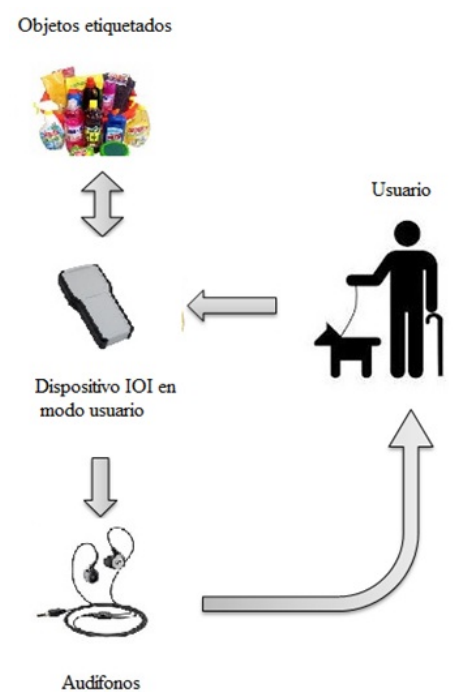

Figura 10. Funciones del usuario dentro del sistema IOI.

\subsection{Pruebas técnicas}

Las pruebas técnicas se realizaron con el objetivo de definir los valores mínimos para el funcionamiento del dispositivo y del software. Los parámetros en los que se basaron las pruebas del dispositivo fueron la duración del suministro de energía, tolerancia a interferencias en la lectura, el número de etiquetas que se puede almacenar y registrar, el ángulo y distancia de cobertura del lector RFID. Por otra parte las pruebas técnicas del software se basaron en la tolerancia a errores, compatibilidad con los sistemas operativos e instalación.

Para definir la duración de los suministros de energía se consideró que el consumo de corriente del dispositivo es de $425 \sim \mathrm{mA}$ cuando está encendido y con todos sus módulos funcionando.

La distancia y cobertura de lectura de las etiquetas se estimó mediante una prueba de medición de ángulo y distancia. La Tabla 1 muestra los resultados de las pruebas técnicas aplicadas al dispositivo IOI.

Para determinar la capacidad de almacenamiento se tomó como referencia que cada archivo de audio tenía un tamaño de $2 \mathrm{MB}$ (una duración aproximada de 2 minutos) y que la capacidad de la memoria microSD era 4 GB.

El software se instaló en los sistemas operativos Windows 7, Vista, XP SP3 y se calculó el tiempo promedio del proceso. También se lo utilizó durante una hora registrándose 30 etiquetas, este proceso no provocó ningún error al sistema operativo que lo alojó. En la tabla 2 se encuentran los resultados de las pruebas aplicadas al software IOI. 


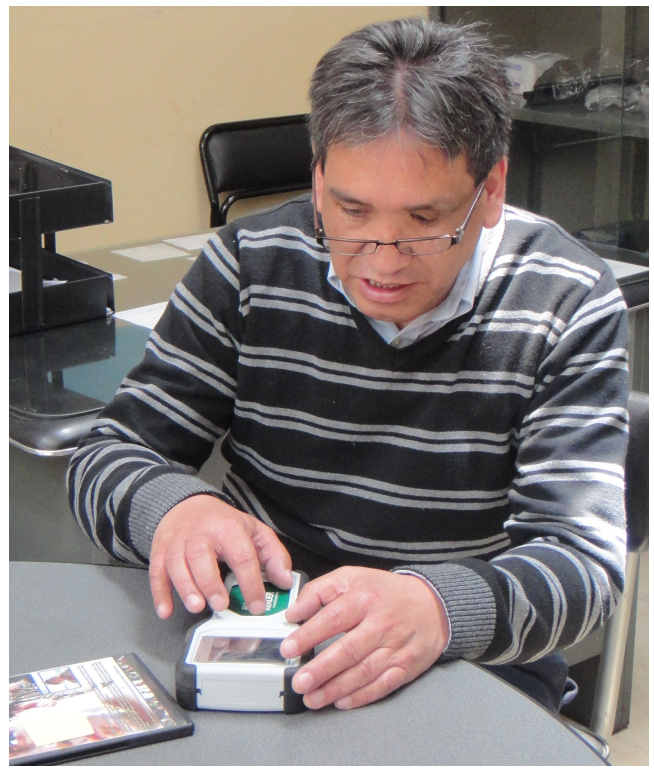

Figura 11. Pruebas realizadas en IEISA.

Tabla 1. Resultados de las pruebas técnicas aplicadas al dispositivo IOI.

\begin{tabular}{cl}
\hline Parámetro & Valor \\
\hline \multirow{2}{*}{ Duración suministros de energía } & Pilas: $\sim 2 \mathrm{~h}$ \\
Capacidad de almacenamiento & Hatería: $\sim \mathrm{h}$ \\
Cobertura del lector RFID & Distancia máxima: $\sim 6 \mathrm{~cm}$ \\
Capacidad de lectura & Ángulo de cobertura: $360^{\circ}$ \\
& Lectura normal ante la presencia \\
& de objetos de papel, plástico, metal \\
& y vidrio. \\
Resistencia & No es resistente al agua, ni a la \\
& humedad. \\
& Puede soportar un peso aproxi- \\
& mado de $30 \mathrm{~kg}$ \\
Dimensiones & $205.3 \times 103 \times 37 \mathrm{~mm}$ \\
Peso & $436,5 \mathrm{~g}$ \\
\hline
\end{tabular}

\subsection{Pruebas de satisfacción}

La estimación del nivel de satisfacción de una persona frente a un dispositivo nuevo se basa en los beneficios y utilidades que este le presenta. El nivel de satisfacción con el dispositivo y software IOI se estimó a través de una encuesta. Los resultados se recopilan en la Tabla 3.

\section{Ajustes}

Luego de las pruebas se determinó subir el volumen en la reproducción de sonido del dispositivo IOI. También se estableció subir el volumen para la grabación y reproducción de archivos MP3 en el software. Otra modificación hecha fue reducir el número de botones y ventanas del software para hacerlo más versátil y fácil de usar; además de incluirle un manual del usuario.
Tabla 2. Resultados de las pruebas técnicas aplicadas al software IOI.

\begin{tabular}{cl}
\hline Parámetro & \multicolumn{1}{c}{ Respuesta } \\
\hline Compatibilidad & Windows 7, Vista y XP SP3 (32 bits) \\
& Windows Installer 3.1 (x86) \\
& Windows Imaging Component (x86) \\
& Windows Installer 4.5 for Windows Vista (x86) \\
Pre-requisitos & Microsoft .NET Framework 2.0 SP2 \\
& Microsoft .NET Framework 3.5 SP1 \\
& Microsoft .NET Framework 4.0 Client \\
& Microsoft SQL Server 2008 Express \\
Soporte & Tiene ayudas para solucionar errores. \\
Tamaño & Con redistribuibles 409 MB \\
& Sin redistribuibles 13,3 MB \\
\hline
\end{tabular}

Tabla 3. Resultados de las pruebas de satisfacción aplicadas para el dispositivo y software IOI.

\begin{tabular}{ll}
\hline \multicolumn{1}{c}{ Parámetro } & Respuesta \\
\hline Nivel de satisfacción con el dispositivo IOI & $9 / 10$ \\
Nivel de satisfacción con el software IOI & $9 / 10$ \\
\hline
\end{tabular}

\section{Conclusiones}

Con el dispositivo IOI es posible que las personas invidentes puedan conocer las características de ciertos objetos, lo que facilita el desarrollo de sus actividades diarias.

El poder conectar el dispositivo IOI a un PC, recibir los datos de una etiqueta RFID, grabar y reproducir archivos MP3 y manejar una base de datos en la que se puede guardar información adicional sobre los objetos etiquetados, permite a los familiares, maestros, dueños de comercios y demás personas administrar el sistema IOI y ayudar a las personas invidentes en la identificación de objetos.

Se espera seguir mejorando con el fin de que personas invidentes puedan acceder al dispositivo y emplearlo sin problemas en tareas de compra y venta en supermercados, comercios, almacenes, etc.

Como trabajo futuro se espera crear un dispositivo único capaz de cubrir la mayoría de las necesidades de los invidentes como identificar billetes, transporte público, vivienda, esquivar objetos, leer libros, etc.

\section{Agradecimientos}

Un agradecimiento especial a todas las personas invidentes de las instituciones SONVA, IEISA y FENCE de la ciudad de Cuenca, quienes aportaron con ideas, entusiasmo y tiempo para que la implementación del sistema IOI fuese posible. 


\section{Referencias}

[1] CONADIS. (2012) Persona registradas en el CONADIS. Ecuador. [Online]. Available: http: //www.conadis.gob.ec/provincias.php

[2] E. Microelectronic Marin SA, EM4100 - Read Only Contactless Identification Device, 2004. [Online]. Available: http://www.smartstripe.com/ pdf/EM4100.pdf

[3] RFid Reader Board, Mikroelektronika, 2012. [Online]. Available: http://www.mikroe.com/ downloads/get/1240/rfid_manual_v100.pdf

[4] Mikromedia for PIC32, Mikroelektronika, 2012. [Online]. Availa- ble: http://www.mikroe.com/downloads/get/1380/ mikromedia_for_pic32_manual_v110b.pdf

[5] Creating first project in Visual TFT, Mikroelektronika, 2012. [Online]. Available: http://www.mikroe.com/downloads/get/1523/ visualtft_ctfp_v200.pdf

[6] MikroC Pro for PIC32 User Manual, Mikroelektronika, 2012. [Online]. Available: http://www.mikroe.com/downloads/get/1608/ mikroc_pro_pic32_v100.pdf

[7] Visual C\# 2010 Express, Microsoft, 2010. [Online]. Available: http://www.microsoft.com/visualstudio/ eng/downloads\#d-2010-express 